\title{
APROXIMACIÓN AL CONCEPTO DE INFERENCIA DESDE DOS MODELOS DE COMPRENSIÓN: MODELO ESTRATÉGICO Y MODELO DE CONSTRUCCIÓN E INTEGRACIÓN*
}

\author{
Carolina Margarita González Ramírez**
}

\begin{abstract}
Resumen
Este trabajo intenta realizar una aproximación al concepto de inferencia a partir de dos modelos de comprensión: modelo estratégico (van Dijk \& Kintsch, 1983) y modelo de construcción e integración (Kintsch, 1988, 1998, 2001). Se trata, por tanto, de observar si este concepto presenta variaciones en su concepción y aplicación en el proceso de comprensión, de acuerdo a los planteamientos de cada modelo. Para tales efectos se realizará un recorrido por las diversas perspectivas (lógica, psicológica y psicolingüística) con el objeto de presentar que se ha entendido por inferencia y luego, se llevará a cabo un estudio comparativo entre los modelos antes señalados respecto del concepto de inferencia que plantea cada uno de ellos, utilizando como criterio de comparación los postulados de la hipótesis minimalista (McKoon \& Ratcliff 1992, 1995) y de la teoría construccionista (Greasser, Singer \& Trabasso, 1994).
\end{abstract}

Palabras clave: Inferencia, modelo estratégico, teoría construccionista.

\section{APPROACH TO THE CONCEPT OF INFERENCE FROM TWO MODELS OF COMPREHENSION: STRATEGIC MODEL AND CONSTRUCTION AND INTEGRATION MODEL}

\begin{abstract}
This paper attempts an approach to the concept of inference from two models of comprehension: strategic model (van Dijk \& Kintsch, 1983) and construction-integration model (Kintsch, 1988, 1998, 2001). It is, therefore, to observe whether this concept presents variations in its design and implementation in the process of comprehension, according to the proposals of each model. For this purpose a journey made by the various perspectives (logical, psychological and psycholinguistic) in order to present has been understood by inference, then, a comparative study was carried out between the aforementioned models on the concept of inference raises each using as a criterion for comparison the fundamental source of the minimalist hypothesis (McKoon \& Ratcliff, 1992; 1995) and the constructionist theory (Greasser, Singer \& Trabasso, 1994).
\end{abstract}

Keywords: Inference, estrategic model, constructionist theory. aprendizaje y procesamiento de reglas sintácticas? Pervivencia e influencia en el rendimiento escolar.

* Chilena. Doctora en Didáctica de la Lengua y la Literatura de la Universidad Autónoma de Barcelona. Académica Pontificia Universidad Católica de Valparaíso, Valparaíso, Chile. carolina. gonzalez.r@pucv.cl 


\section{Introducción}

La comprensión constituye uno de los procesos más complejos mediante el cual adquirimos conocimiento. Es posible considerar como un hecho altamente consensuado el que la comprensión de un texto no se realiza completamente a partir de la información explícita que éste provee, sino que se alcanza a través de la reconstitución que realiza el lector de la información implícita necesaria para la elaboración de una representación coherente del texto por medio de inferencias.

Las inferencias permiten añadir, sustituir, integrar $\mathrm{u}$ omitir información necesaria para que el lector asigne coherencia al texto, tanto local como global. Es por este motivo que algunos autores destacan el lugar central que las inferencias deben ocupar el en el estudio de la comprensión (León, 2003; Parodi, 2005).

Si bien es clara la importancia que tiene la inferencia, no existe acuerdo en cómo se concibe, y menos en cuáles son los procesos inferenciales que realizan los lectores, ni cómo y cuándo operan. Es por este motivo que el concepto ha sido abordado desde diversas perspectivas (lógica, psicológica y psicolingüística). Pese a la diversidad de perspectivas es posible encontrar rasgos que constituirían lo esencial del concepto inferencia, puesto que las perspectivas no son excluyentes.

Este trabajo pretende dilucidar cuál es el concepto de inferencia que subyace a dos modelos de comprensión: Modelo estratégico (van Dijk \& Kintsch, 1983) y Modelo de construcción e integración (Kintsch, 1988, 1998, 2001). Para dicho propósito, se expondrán algunas de las definiciones de inferencia desde diversas perspectivas; posteriormente se presentarán los aspectos más relevantes de cada uno de los modelos, destacando sus fortalezas y debilidades, con el objeto de realizar el análisis comparativo en cuanto al tema que nos compete utilizando como criterio de comparación dos propuestas teóricas: la hipótesis minimalista (McKoon, \& Ratcliff, R, 1992, 1995) y la teoría construccionista (Graesser, Singer, \& Trabasso, 1994).

\section{Concepto de inferencia}

En las diversas definiciones de inferencia es posible encontrar rasgos comunes que permiten, de modo general, identificarlas como tales. Sin 
embargo, algunas definiciones incluyen o resaltan rasgos que evidencian un mayor énfasis en algún aspecto particular. Cada una de estas definiciones se inserta en una perspectiva determinada, proporcionando una visión particular de inferencia.

Desde la lógica formal la inferencia ha sido entendida según Bunge (1960) «el paso de un conjunto de proposiciones a otro; el primer conjunto puede llamarse la clase de premisas y el segundo la clase de conclusiones» (p. 860). Esta definición apunta a la inferencia como una estructura silogística, es decir, una entidad lógica de tres niveles: dos premisas y una conclusión. Por su parte, Ferrater Mora (1980) concibe la inferencia como «conjunto de todos los procesos discursivos» (p. 860) distinguiendo entre procesos inmediatos y procesos mediatos. A partir de estos procesos se generan las llamadas inferencias inmediatas, que consisten básicamente en la conclusión de una proposición sin la intervención de una tercera. Por otra parte, se encuentran las inferencias mediatas, en las cuales la conclusión de una proposición se obtiene por medio de la intervención de una $\mathrm{u}$ otras proposiciones.

Esta perspectiva, se caracteriza por prestar atención a los aspectos formales de la inferencia, básicamente lo sintáctico. Los principios lógicos y sintácticamente correctos son válidos en cuanto nivel formal, pero no en términos de verdad o falsedad de las premisas. Parodi (2005) hace mención a la identificación entre la lógica formal y los procesos mentales de razonamiento humano, puesto que desde ese punto de vista, se considera que el razonamiento humano opera en base a reglas lógicas de inferencias, otorgando al pensamiento un carácter deductivo. Pese a que, es posible considerar procesos lógicos tanto deductivos como inductivos, la psicología y la lingüística se han limitado a lo deductivo. Por tanto, desde esta concepción la inferencia sería generada desde la relación entre las premisas (relaciones sintácticas) y no por la relación que podría establecerse con la realidad.

Situándonos en la perspectiva psicológica, el concepto de inferencia cambia. Se considera la veracidad como un criterio de evaluación de este proceso para determinar si es válido. Debido a este motivo, es que el interés de esta perspectiva radica principalmente en el aspecto semántico. En este contexto, importan los contenidos de las premisas, puesto que la lógica no puede determinar si todas las conclusiones que los lectores realizan de manera espontánea son válidas. 
Es posible considerar la inferencia como deductiva e inductiva, ya que podemos generar información utilizando uno u otro procesamiento. La importancia de la inferencia radica en la producción de un nuevo conocimiento, facilitando la comprensión.

Johnson-Laird (1986) señala que es posible realizar un razonamiento sin lógica para construir inferencias válidas. La validez estaría dada por principios semánticos (la relación de los contenidos), y no por reglas lógicas. Este tipo de procesamiento es heurístico (De Vega, 1984); el lector busca estrategias conceptuales y asociativas para elaborar una representación mental que incluye el contexto en el cual está inserto, construyendo así diversos procesos inferenciales.

Ya hemos visto que la conceptualización sobre inferencias es un tema no zanjado. En el marco de las perspectivas posibles, consideramos que los aportes realizados por la psicolingüística son de vital importancia para este trabajo. Según lo planteado por Parodi (2005) la inferencia es un: «Conjunto de procesos mentales que -a partir de la información textual disponible y la correspondiente representación mental coherente elaborada por quien lee- un sujeto realiza para obtener un conocimiento nuevo no explicitado, toda vez que se enfrenta a la comprensión de un texto dado» (p. 51).

Los procesos mentales involucrados en la generación de inferencias están vinculados a la activación y recuperación de información. La activación se produce debido al estímulo producido por la información que el texto provee y que es la señal para la recuperación de la información desde la memoria a largo plazo (MLP). Tanto la información entrante como la recuperada desde la MLP, confluyen para elaborar una representación coherente del texto (León, 2003).

Esta perspectiva se centra en el aspecto pragmático, puesto que las inferencias se realizan en el ámbito de la comprensión. No está presente la idea de razonamiento lógico deductivo, pero esto no significa que el lector no utilice silogismos para resolver problemas de comprensión.

La inferencia se realiza dentro de un contexto determinado; se construye a partir de la información cognitiva que el sujeto lector, almacena producto de sus experiencias y conocimiento de mundo. Este proceso puede ser realizado durante o después de la comprensión, ya que, en uno $\mathrm{u}$ otro momento son sustentadas por el conocimiento previo del 
sujeto. La consideración del momento en que se realizan las inferencias y de qué tipo son, darán origen a dos posturas teóricas. Tales propuestas se conocen como hipótesis minimalista, planteada por Mckoon y Ratcliff (1992, 1995) y teoría construccionista de Graesser, Singer y Trabasso (1994).

\section{Hipótesis minimalista}

La hipótesis minimalista fue planteada por McKoon y Ratcliff (1992, 1995) en respuesta a la difundida teoría construccionista, que en sus comienzos se ubicaba en una posición bastante extrema, ya que consideraba que todas las conexiones de un mensaje son viables y pueden ser ejecutadas durante la comprensión, una posición totalmente maximalista, que ha sido cuestionado por los construccionistas posteriores: «One of the shortcomings of early constructionist theories [...] is that failed to make specific predictions about the inferences and meaning representations that are constructed during encoding» (Graesser et al., 1994, p. 371).

Según el planteamiento de la hipótesis minimalista, en el proceso de la comprensión interviene información proveniente de dos fuentes: el contenido explícito del texto y el conocimiento del lector. Éstas interactúan para conformar la representación del texto y es en esa interacción donde se producen las inferencias, que son bastante restringidas dentro de este enfoque, ya que se limita solo a aquéllas que se producen de manera automática durante el proceso de encodificación, donde el lector no se guía por metas específicas:

The minimalist hypotesis describes the kinds of inference that will be generated during reading when the readers engages in no special efforts, goals, or strategies or problem-solving kinds of texts. In the absence of such strategies, the minimalist hypothesis states that only two kinds of inferences will be generated during reading: those based on easily and quickly available information and those requiered to establish local coherence. (McKoon \& Ratcliff, 1995, p. 99).

Según la hipótesis minimalista, los criterios que determinan la realización de inferencias son dos: la facilidad y rapidez de acceso a la información desde la memoria y el establecimiento de la coherencia local. 
Desde esta perspectiva surge una distinción respecto de las inferencias, que las divide en automáticas y estratégicas. Las primeras, inferencias automáticas, son construidas durante la lectura sin la intervención de procesos estratégicos ni mediación de la conciencia. Su función radica en establecer la coherencia local (León, 2003). La información a la que se debe tener fácil acceso es aquella que proviene del texto y que se encuentra disponible en la memoria a corto plazo, o bien, se basa en un conocimiento muy general y bien conocido. En esta perspectiva, el énfasis está puesto en la accesibilidad de la información y no en la fuente de que ésta proviene.

\subsection{Inferencias automáticas}

A la luz de los criterios señalados anteriormente los autores reconocen como automáticas, solo las inferencias puente y las inferencias causales antecedentes. Las primeras son aquellas que establecen la unidad proposicional a través de la conexión entre elementos léxicos o por medio de anáfora; este tipo de inferencia cumple con las condiciones necesarias de automaticidad en cuanto a la rapidez con que son realizadas (McKoon \& Ratcliff, 1995).

Por otra parte, las inferencias causales conectan tanto la información proveniente del texto con la proporcionada por los conocimientos previos del lector, por tanto, su función es establecer relaciones causales locales entre la información que se está leyendo y la que proviene de los conocimientos del lector. Si bien, los autores consideran que estas inferencias son realizadas durante la lectura, es necesario precisar qué inferencias causales son necesarias para establecer la coherencia.

\subsection{Limitaciones de la hipótesis minimalista}

La hipótesis minimalista no considera la mayor parte de las inferencias que son necesarias para la elaboración de un modelo de situación. Los autores señalan que las inferencias automáticas son desarrolladas para establecer la coherencia local del discurso, pero las inferencias globales, que conectan segmentos de texto distantes, no son realizadas de manera automática. El énfasis puesto en las inferencias automáticas excluye inferencias de otro tipo, como las de carácter elaborativo, entre las que se incluyen las inferencias instrumentales, y las predictivas, puesto que los autores consideran que es necesario utilizar ciertas estrategias para 
realizarlas, puesto que requieren de más procesos cognitivos, lo que las hace más lentas (León, 2003).

\section{Teoría construccionista}

La teoría construccionista planteada por Graesser, Singer y Trabasso (1994) sostiene que la representación mental de un texto se corresponde con el modelo de situación que éste describe. En este contexto, señalan que «an adequate psychological theory of the text comprehension should be able to account for the generation of inferences when readers construct a situation model of what a text about» (p. 371).

La teoría plantea que las inferencias basadas en el conocimiento pueden ser realizadas durante la comprensión, aunque se distinguen aquellas que precisamente son generadas durante el proceso de lectura, que han sido denominadas on-line, de aquellas que se realizarían de manera posterior en una fase elaborativa, inferencias off-line.

A diferencia de la teoría minimalista, ya no es el automatismo con que se realicen o no las inferencias el criterio diferenciador, sino que ahora toma relevancia la posibilidad de que se hagan durante o después de la lectura. Las metas del lector no se utilizan como un elemento diferenciador, puesto que se considera que éste siempre procesa orientado hacia una meta.

La teoría construccionista tiene como principio fundamental «Searchafter-meaning» (p. 371), el cual es explicado a través de tres supuestos básicos:

1) Supuesto de la meta del lector: la representación construida por el lector siempre está dirigida por una meta.

2) Supuesto de coherencia: los lectores intentan elaborar una representación que sea coherente tanto a nivel local como global.

3) Supuesto de explicación: los lectores intentan explicarse el porqué de las acciones y estados que son mencionados en el texto.

A diferencia de la hipótesis minimalista, la teoría construccionista afirma que las inferencias se realizan en la construcción del modelo de situación, por tanto, pretenden dar cuenta de una comprensión profunda, la cual se hace efectiva cuando el lector es capaz de construir 
una explicación de la relación entre los eventos presentes en el texto. Es por este motivo que las inferencias basadas en el conocimiento adquieren una vital importancia; estas inferencias se construyen cuando se activa el conocimiento almacenado en la memoria de largo plazo.

Las metas del lector orientan la elaboración de un modelo de situación, y ahí radica su importancia. Es posible distinguir tres niveles de metas: el primer nivel está constituido por la meta del lector, que consiste en la construcción de un modelo de situación acorde al texto; en el segundo nivel, las metas están asociadas al género del texto: entretención si se lee una novela, informativo al leer un artículo científico, y finalmente, en el tercer nivel, se encuentran las metas particulares y específicas del lector, qué busca con su lectura (Grasser et al., 1994).

\subsection{Inferencias on-line y off-line}

La teoría construccionista plantea seis tipos de inferencias que se realizarían de manera on-line (Graesser et al.,1994), es decir durante el proceso de lectura: referenciales (inferencias pronominales), asignación de rol, antecedente causal (motivos o razones que permiten explicar eventos), meta principal, temática (determinar cuál es el tema del texto que estoy leyendo), reacción emocional del personaje.

Dentro de las inferencias off-line (Graesser et al.,1994), realizadas en una fase posterior a la lectura, están las siguientes: consecuencia causal, sustitución de categoría por ejemplar, instrumento, acciones y metas subordinadas, emocionalidad del lector y propósito del autor.

\subsection{Limitaciones}

Esta división es restrictiva y en algunos casos errónea, puesto que en algunas inferencias consideradas como off-line es posible realizarlas durante el proceso de lectura, las inferencias que dicen relación con ubicación de objetos espaciales y la sustitución de categorías. A la inversa también, inferencias consideradas on-line, son realizadas después de la lectura, puesto que involucran estrategias y ciertos procesos cognitivos por parte del lector.

Otro aspecto criticable, es que muchas de las inferencias que propone son propias de los textos narrativos (León, 2003) lo que limita el campo de realización, puesto que no es posible aplicar la totalidad del modelo a 
diversas tipologías textuales. El que la teoría construccionista realice su teorización en base a textos narrativos, está asociado al planteamiento de la construcción de un modelo de situación del texto, que se ve favorecido por el parecido que se da entre las experiencias de la vida cotidiana con las presentadas en los textos narrativos.

\section{Modelos de comprensión e inferencias}

\subsection{Inferencias en el modelo estratégico de van Dijk y Kintsch (1983)}

Este modelo es de carácter estratégico, es decir, el procesamiento de la información es interactivo y no está restringido por reglas, lo que otorga mayor flexibilidad permitiendo el uso de estrategias por parte del lector. Destaca su carácter descendente, puesto que los conocimientos previos del lector cumplen un rol esencial en la comprensión.

Los autores van Dijk y Kintsch (1983) señalan que, «a strategy is merely a global instruction for each necessary choice to be made along the path of the course of action: whatever happens, always choose the cheapest, fastest, surest, etc., alternative» (p. 64-65).

Por otra parte, sostienen que «that estrategy is a cognitive representation of some kind, that it pertains to complex action sequens that it is linked to the notion of a plan» (p. 65). Las estrategias son procedimientos cognitivos individuales, puesto que le permiten al lector hacer uso de sus propios conocimientos así como también le es posible utilizar de una manera flexible la información entregada por el texto (Parodi, 2003). Este cambio de estrategias por reglas repercutió en la visión que el modelo plantea respecto de la macroestructura.

Dado que en el Modelo deSituación(1978) se plantea el funcionamiento a base de reglas, se consideraban las macroreglas, que en el modelo actual pasan a constituir las llamadas macroestrategias, las cuales, al igual que en el modelo anterior, se realizan bajo el control de un esquema, pero ahora se especifica que el esquema se orienta hacia el cumplimiento de metas y objetivos del lector.

El modelo ha sido considerado psicosociolingüístico, ya que incluye dos supuestos básicos: uno cognitivo y otro contextual. Cada uno engloba 
una serie de supuestos que contribuyen a la conformación del supuesto principal. La noción de supuestos contribuye a la consideración de que el discurse se produce en un contexto social.

Respecto del supuesto cognitivo, podemos señalar que cada vez que un lector realiza una lectura construye una representación mental de la comprensión, y para ello debe considerar sus conocimientos previos respecto del tema y la información que el mismo texto le entrega. En cuanto al supuesto contextual, se plantea que la comprensión se produce dentro de una situación particular y en un contexto sociocultural.

El modelo es dinámico, van Dijk \& Kintsch (1983) señalan: «the interaction in which the processing of discourse is embedded is itself part of a social situation» (p. 7). Las unidades que lo componen están en una constante interacción. Así la información textual y contextual se conecta con la información interna que el lector posee, actuando de manera paralela. Un aspecto relevante de este modelo es que propone un nuevo nivel de representación que se suma a los ya existentes en 1978 (código de superficie y base textual) el Modelo de Situación, el cual comprende una representación episódica de la situación descrita en el texto. Para la construcción de un Modelo de Situación se debe considerar la información proveniente de la memoria episódica, en donde se almacenan las experiencias del sujeto, los esquemas y guiones que éste ha ido formando a partir de la adquisición de conocimiento. Al respecto, los autores señalan:

the situation model that is constructed in this way is the basis for the interpretation of the text. It features all the knowledge that is left implicit in the text or otherwise presupposed. General knowlegde is treated exactly like specific experiences in the respect: both may form basis for situation models, and hence for the encoding of new experiences. (p. 337).

La inclusión de este nivel de representación se justifica porque consideran que es esencial para el establecimiento de la referencia. Los autores plantean que: «The world, as it is referred to, is not cognitively relevant: what we see or think about is again some construction, namely, the situation model» (p. 338). Esto permite llenar aquellos vacíos que eventualmente podrían generarse mientras se lleva a cabo el proceso de comprensión. 
Por su parte, el modelo estratégico posee múltiples niveles de procesamiento y de representación, lo que se coordinan en tiempo real, es decir on-line. La MLP que contiene el conocimiento general se activa en el procesamiento, así como las metas que orientan la interpretación que el lector realiza al elaborar un modelo de situación, utilizando procesos inferenciales.

Van Dijk y Kintsch (1983) señalan que la inferencia es un proceso esencial en la comprensión del discurso. Consideran además que un aspecto distintivo de las inferencias realizadas en la base textual, es el grado certeza con que se las puede realizar, puesto que la información se desprende del texto mismo: «one aspect that distinguishes text-based inferences is the degree of certainty with which they can be made. Some inferences appear to be necessary consequences of the text, whereas others we are less certain about and still others we would regard as no more than plausible or possible conjectures.» (p. 48).

Además, realizan una conceptualización de la inferencia a partir de una inquietud general: «the biggest problem with discourse inferences is to determine when they are made» (p. 48).

Esto se asocia a lo planteado por la teoría construccionista, ya que se produce una dicotomía, que en algunos casos es errónea, en relación al momento en que se produce la inferencia, si es on-line u off-line (Graesser et al., 1994). Esta preocupación ha sido compartida por la perspectiva psicolingüística, puesto que resulta difícil determinar en qué momento se realizan las inferencias, y de qué tipo son.

Sin la intención de realizar una taxonomía de inferencias los autores distinguen dos tipos; por una parte están las llamadas inferencias puente, «one class of inferences that appear to the necessary during comprehension are the bridging inferences requiered for the coherence of the text.» (van Dijk \& Kintsch, 1983, p. 49). Según los autores estas inferencias se desprenden del texto mismo, en este sentido las inferencias léxicas serían plausibles y no presentarían dificultades en su identificación, puesto que no implicarían grandes procesos cognitivos.

Por otra parte, las inferencias elaborativas, se llevarían a cabo en una instancia posterior a la lectura: «elaborative inferences occur when the reader uses his or her knowledge about the topic under discussion to fill in additional detail not mentioned in the text, or to establish connections 
between what is being read and related items of knowledge» (p. 50). $\mathrm{Si}$ bien, este tipo de inferencias no es necesariamente realizadas durante la lectura, cuando se llevan a cabo aportan beneficios a la recuperación de información desde la memoria. Esto se debe al carácter descendente del modelo, puesto que se presta atención a los conocimientos previos del lector. Un punto esencial de este modelo es que concibe la base textual como una representación del texto a nivel proposicional y sitúa a las inferencias puente y a los otros tipos dentro del modelo de situación, lo que implica un desplazamiento de la inferencia desde un nivel lingüístico a un nivel conceptual.

Del mismo modo que la teoría construccionista (Graesser et al., 1994), el modelo estratégico sitúa las inferencias en el modelo de situación, por tanto se aboga por una comprensión profunda, en donde tienen mayor cabida los conocimientos previos del lector para la elaboración de inferencias, utilizados de manera estratégica, es decir, el sujeto se enfrenta al texto y ejecuta tareas que se asocian a sus objetivos como lector, eso justifica el uso de estrategia, todo esto con el objeto de elaborar una representación mental del texto de manera coherente, tanto a nivel local como global.

Es preciso mencionar que los autores sientan las bases para los planteamientos posteriores que se establecen el nivel de representación correspondiente al modelo de situación para la consideración de las inferencias que pueden ser realizadas.

\subsection{Inferencias en el modelo de construcción e integración de Kintsch $(1988,1998,2001)$}

El modelo de construcción e integración es considerado híbrido, puesto que combina posturas extremas; por una parte la construcción es de carácter simbólico y está basado en reglas, mientras que en el proceso de integración hay una base conexionista. La primera fase del modelo, construcción, es de carácter ascendente, puesto que la información del texto guía el proceso de comprensión. La segunda fase en tanto, integración, es descendente, en donde los conocimientos previos del lector tienen un papel crucial.

El modelo presenta estados de avance, puesto que se ha ido desarrollando y ampliando en una secuencia que tiene por objetivo desarrollar una teoría general sobre la cognición. Kintsch(1998) señala que: 
«the theory presented here is a proposal for an architecture of cognition. that is, it is a collection of specific models, all employing the same general architectural framework» (p. 5). Esta arquitectura se organiza en base al conexionismo, que mantiene la idea de representación, no de un modo simbólico, sino a través del estado de una red conexionista en que se distribuye el conocimiento por los pesos de las conexiones.

Si bien, el modelo de construcción e integración reconoce la existencia de varios niveles de representación, esto es, código de superficie, base textual y modelo de situación, pone un marcado énfasis en la constitución de la base textual en el proceso de construcción: «It combines a construction process in which a text base is constructed from the linguistic input as well as from the comprehender's knowledge base, with an integration phase in which this the text base is integrated into a coherent whole. The knowledge base is conceptualized as an associative network» (p. 163).

El proceso que considera el modelo comienza con la construcción de una red asociativa de elementos:

The steps in constructing a text base according lo the construction-integration model involve: (a) forming the concepts and propositions directly corresponding to the linguistic input. (b) elaborating each of these elements by selecting a small number of most closely associated neighbors from the general knowledge net; (c) inferring certain aditional propotions; and (d) assigning connection strengths to all pairs of elements that have been created. (p.165).

Luego se avanza hacia una fase de integración de carácter conexionista en que se elabora una representación del texto que excluye los elementos irrelevantes.

Considerando el enfoque conexionista que presenta el modelo, se asume que al pasar de una unidad a otra en el procesamiento, parte de lo procesado no se desactiva en la memoria de trabajo. Estas proposiciones se mantienen focalizadas y se procesan junto a las proposiciones que pertenecen al siguiente ciclo de procesamiento. De este modo, es que en el proceso de construcción se desarrolla una red de proposiciones interrelacionadas. En el proceso de integración, a las proposiciones se les asigna un valor de fuerza de activación y a través de estos valores, la red 
alcanza un estado de equilibrio en la memoria episódica, propiciando la actualización de la información de la memoria de largo plazo.

En cuanto a las inferencias podemos señalar que el modelo concuerda con la hipótesis minimalista, en cuanto esta asigna importancia a la información proporcionada por el texto mismo para la construcción de inferencias asociadas a la coherencia local, de esta manera el proceso sería netamente ascendente. El modelo de construcción e integración contempla la posibilidad de añadir inferencias en la representación del texto, lo cual se realiza a través del incremento en la fuerza de las conexiones asociadas al significado contextualmente más apropiado, es decir el proceso inferencial adecuado la que hacíamos mención en el proceso de construcción, que se realiza a partir de la información que es de más fácil y rápido acceso. Este hecho significa un respaldo para los minimalistas quienes consideran que: «the inference could only be generated from information that is quickly and easily available»(McKoon \& Ratcliff, 1995, p. 103).

Pese a esto, los minimalistas reconocen que el modelo de construcción e integración, contempla la realización de otras inferencias que no están basadas en la información inmediatamente disponible, como las inferencias basadas en el conocimiento.

Kintsch (1998) realiza una clasificación de las inferencias en base a dos criterios básicos: la recuperación de información inmendiatamente disponible en la memoria y la generación de información a partir de lo que el texto propone. Estas inferencias contribuyen a la construcción de un modelo de situación.

Desde los dos criterios planteados por el autor para determinar las inferencias, los tipos que plantea son los siguientes: inferencias puente, e inferencias transitivas, que en definitiva se constituyen por una asociación de información. Son procesos automáticos, puestos que involucran recuperación de información; según el autor no generan nuevo conocimiento y serían realizadas durante la lectura, ya que son elementales para el mantenimiento de la coherencia local (Kintsch, 1998). Este tipo de inferencias requiere menor tiempo para su ejecución, por tanto son automáticas.

El otro tipo de inferencias que distingue el autor, corresponden a procesos controlados, puesto que el sujeto debe hacer uso de estrategias 
para obtener el conocimiento. Las inferencias a las que hacemos referencia son las denominadas "puente basadas en la búsqueda de conocimiento» y las inferencias lógicas. Las primeras involucran procesos de recuperación de información almacenada en la memoria de largo plazo, por tanto necesitan más tiempo para su elaboración. Respecto de las inferencias lógicas, cabe señalar que generan conocimiento, y son consideradas una representación mental abstracta que en algunos casos está basada en reglas.

Es importante señalar que pese a que el modelo de construcción e integración concuerda con la hipótesis minimalista respecto de las inferencias que son necesarias para establecer la coherencia local, no comparte la idea de que sean solo este tipo de inferencias que se realiza durante la lectura, ya que considera que aquéllas que contribuyen a establecer la coherencia global, (inferencias temáticas, inferencias asociadas a la emocionalidad e inferencias de meta principal, Graesser et al., 1994 ) también pueden ser elaboradas dentro del proceso de lectura y no de manera posterior como lo sugieren los minimalistas.

Como mencionamos anteriormente, el modelo contempla las inferencias basadas en el conocimiento o elaborativas, pero no realiza una taxonomía, puesto que estas inferencias demandan mayor cantidad de tiempo en su elaboración, y no están basadas en la información inmediatamente disponible, por ende no se llevan a cabo durante la lectura, si no que más bien, se realizarían de manera posterior.

Según Kintsch (2001), las inferencias causales son de orden pragmático, y podrían considerarse dentro de las inferencias basadas en el conocimiento, ya que la intervención de los conocimientos previos del lector es bastante relevante. Estas inferencias pueden ser comprendidas a partir de la aplicación de LSA (Análisis semántico latente), que permite la relación entre proposiciones que se encuentran de manera más distante el texto.

Es preciso señalar que dentro del modelo de construcción e integración se da relevancia a las inferencias puente, puesto que estas permiten la elaboración de una representación mental coherente, que da paso a la construcción de un modelo de situación, en donde se funden la información nueva con aquélla que está almacenada en la memoria. 


\section{Conclusiones}

El concepto de inferencia no es un tema totalmente delimitado, esto se puede explicitar con el hecho de que aún no se ha realizado un modelo teórico general que abarque esta problemática.

Si bien los modelos presentados, modelo estratégico (van Dijk \& Kintsch, 1983) y modelo de construcción e integración (Kintsh, 1988, 1998, 2001) no plantean una clara definición de inferencia, sí realizan una conceptualización, es decir, formulan planteamientos sobre como ésta es representada en el proceso de comprensión y la incidencia que esta tiene en dicho proceso, esto puede ser apreciado en los tipos de inferencias que establece cada modelo.

Existe un consenso entre el modelo estratégico y el modelo de construcción e integración, respecto de las llamadas inferencias puente, las que según los planteamientos de dichos modelos, se realizarían durante el proceso de lectura, es decir on-line (Greasser et al., 1994) contribuyendo al establecimiento de la coherencia local.

La relación que se logro establecer entre los modelos y las teorizaciones sobre la inferencia permitió centrarse en los tipos de inferencia que postula cada uno de los modelos en base a determinados criterios.

El modelo estratégico, concuerda con los planteamientos de la teoría construccionista, en tanto privilegia la utilización de conocimientos previos del lector y estrategias que tienen relación con el objetivo que se pretende lograr con la lectura.

Por otra parte el modelo de construcción e integración que en su fase primera es ascendente, lo que implica que se le reste importancia a los conocimientos del lector, privilegiando las inferencias guiadas por el contenido del texto, sustenta a la hipótesis minimalista, ya que considera que las inferencias puente son las que se realizan durante la lectura son pocas y automáticas, es decir la información utilizada para llevar a cabo las inferencias debe ser de fácil acceso.

Es importante señalar que el modelo de construcción e integración plante la inclusión de algunas inferencias que permiten establecer la coherencia global, son también realizadas durante la lectura. Esto no quiere decir que sean totalmente automáticas, ya que requieren de 
mayor tiempo para ser realizadas. Esto afecta la propuesta de la hipótesis minimalista ya que de ser consideradas durante la lectura, las inferencias que se efectuarían no serían mínimas.

En el modelo estratégico de van Dijk y Kintsch (1983) se da relevancia al modelo de situación, en donde ubican las inferencias, justificando esta elección por la amplia gama de concexiones que establece con otros elementos del sistema de procesamiento y por su alto nivel explicativo. En tanto, el modelo de conexión e integración de Kintsch (1988) se da realce nuevamente a la base textual entendida como la elaboración de un significado basado en el conocimiento previo y en la información del texto, por tanto, es formadora de coherencia. Finalmente, cabe señalar que las inferencias no se ubican la base textual, sino más bien en el modelo de situación, ya que son parte de la representación mental que elaboramos a partir de la comprensión.

\section{Referencias bibliográficas}

Bunge, M. (1969). La investigación científica. Barcelona: Ariel.

Ferrater Mora, J. (1980). Diccionario de filosofía. Madrid: Alianza

Gómez Macker, L. \& Peronard, M. (2005). El lenguaje humano. Valparaíso: Ediciones Universitarias de Valparaíso.

Graesser, A., Singer, M. \& T. Trabasso. (1994). Constructing inferences during narrative text comprehension. Psychological Review, 48, 163-189.

De Vega, M. (1984). Introducción a la Psicología cognitiva. Madrid, Alianza Editorial.

Johnson-Laird, P. (1986). Reasoning without logic. Reasoning and discourse processes. London, Academic Press.

Kintsch, W. (1988). The role of knowlegde in discourse comprehension: A construction-integral model. Psychology Review. Colorado: University of Colorado.

- (1998). Comprehension: A paradigm for cognition. New York: Cambridge University Press.

. (2001). Predication. Cognitive Science, 25, 173-202. 
León, J. (2003). Conocimiento y discurso. Claves para inferir y comprender. Madrid: Pirámide.

McKoon, G. \& Ratcliff, R. (1995). The minimalist hypothesis: directions for research. En Ch. Weaver, S. Mannes, \& Ch. Fletcher (Eds.), Discourse comprehension. New Jersey: LEA.

Parodi, G. (1989). La inferencia una aproximación al concepto. En Actas del $8^{\circ}$ Seminario de la Sociedad Chilena de Lingüística (pp. 211-220). Santiago.

(2003). Relaciones entre lectura y escritura: una perspectiva cognitiva discursiva. Antecedentes teóricos y resultados empiricos. Valparaíso: Ediciones Universitarias de Valparaíso.

__. (2005). Comprensión de textos Escritos. Buenos Aires: EUDEBA.

van Dijk, T. \& Kintsch, W. (1983). Strategies of discourse comprehension. New York: Academic Press. 